\title{
Globe
}

Revue internationale d'études québécoises

\section{Cowboy de Louis Hamelin. Le Far West québécois ou la redéfinition des frontières nordiques}

\section{Paola Ruggeri}

Volume 1, numéro 2, 1998

URI : https://id.erudit.org/iderudit/1000510ar

DOI : https://doi.org/10.7202/1000510ar

Aller au sommaire du numéro

\section{Éditeur(s)}

Globe, Revue internationale d'études québécoises

ISSN

1481-5869 (imprimé)

1923-8231 (numérique)

Découvrir la revue

Citer cet article

Ruggeri, P. (1998). Cowboy de Louis Hamelin. Le Far West québécois ou la redéfinition des frontières nordiques. Globe, 1(2), 9-27.

https://doi.org/10.7202/1000510ar

\section{Résumé de l'article}

Cet article fait partie de ma thèse de doctorat, une étude concernant l'image de l'Amérindien dans le roman québécois contemporain. Dans le corpus que j'ai étudié, les Autochtones sont voués à la disparition, même quand ils arrivent à établir des contacts positifs avec les Francophones, car ils représentent un danger pour la société des colonisateurs. J'ai aussi remarqué qu'en général les Indiens ne sont presque jamais au premier plan. Leur importance dans l'intrigue dépend de leurs relations avec les personnages blancs. Cela nous amène à croire que les Autochtones aident ces derniers à se définir, à se construire une identité. Comme cette quête d'identité est étroitement liée à la conquête du territoire, il est aussi question de survie. Dans le roman de Louis Hamelin, les Francophones voudraient s'enrichir par le biais de la création d'un village touristique, Grande-Ourse, où ils invitent des riches États-uniens à pratiquer la chasse et la pêche. Les Amérindiens, qui n'ont aucun intérêt pour ce genre d'entreprise, sont obligés de se déplacer vers le nord du territoire. Ils abandonnent leurs terres à l'exploitation des Blancs et leur société, qui est déjà corrompue, finit par disparaître : un trou noir va absorber leur énergie vitale et de nouvelles frontières vont être tracées dans le nord du Québec. 


\title{
Cowboy de Louis Hamelin Le Far West québécois ou la redéfinition des frontières nordiques
}

\author{
Paola Ruggeri \\ Université de Bologne
}

Si, dans la littérature et le cinéma des États-Unis, le mythe du Far West fonde une dialectique de la frontière en tant qu'espace conflictuel séparant la nature de la culture, et donc les Amérindiens des Blancs, dans le cas du Québec ce sont les vastes solitudes nordiques qui constituent un repère géographique essentiel pour la rencontre de l'autre, l'Autochtone.

L'expansionnisme français en Amérique du Nord découle notamment de l'esprit d'aventure de ces hommes qu'on nommait "coureurs des bois», qui décidaient de partir pour les «Pays d'en haut» pour se libérer des contraintes de la colonie, en quête d'une communion profonde avec la nature et les grands espaces. Au lieu de s'établir définitivement sur un territoire pour le cultiver, ils préféraient une vie nomade, qui leur permettait d'entrer en contact avec les Autochtones dans le cadre de la traite des fournures. Leur conduite n'était pas tout à fait orthodoxe, les activités illégales et le trafic d'alcool étant souvent au centre de leurs commerces, mais leurs déplacements étaient essentiels pour explorer le territoire et pour établir des alliances militaires avec les Amérindiens. Cet appel du Nord, qui s'oppose nettement à la sédentarité des colons établis autour du

1 Montréal, XYZ, 1992, abrégé en CB.

Paola Ruggen, «Cowboy de Louis Hamelin. Le Far West québécois ou la redéfinition des frontières nordiquess), Globe. Revne intermationale d'études queberoises, vol. 1, no 2, 1998. 
Saint-Laurent, est très bien défini par Jack Warwick qui résume ainsi ses caractéristiques principales :

[...] la révélation exaltante du voyage, l'émerveillement devant la diversité des paysages, la nostalgie pour l'homme. J'étais de nature, la démangeaison de bâtir des empires, tout cela constitue une somme d'expériences communes dont fait état la littérature et où il arrive qu'elle trouve ses archétypes. ${ }^{2}$

La nordicité, étroitement liée à l'indianité, est sûrement un des thèmes propulseurs du roman Cowboy de Louis Hamelin, qui semble cependant en démentir quelques aspects. En effet, on voit émerger de cette narration une image de l'Amérindien très différente de celle du «bon sauvage», et le petit empire d'exploitation forestière établi par les Blancs, n'ayant pas de fondements, s'écroule tragiquement.

Les événements racontés se déroulent à Grande-Ourse, un petit village au nord de Montréal, près de Sans-Terre et Tocqueville. Le nom même de cet endroit fictif est un indice de nordicité, car $\mathbf{1}$ fait songer à la constellation principale de l'hémisphère septentrional. Grande-Ourse n'est pas un village comme les autres : en tant qu'avant-poste de colonisation, la zone a été planifiée dans les moindres détails ${ }^{3}$ et la seule loi qui

2 Jack Warwick, L'Appel du nord dans la littérature canadienne-française, Montréal, Hurtubise HMH, 1972, pp. 21-22.

3 «La Compagnie avait coupé le plus qu'elle avait pu, puis elle avait foutu le camp. Ce n'était plus rentable d'opérer à partir de GrandeOurse. Elle avait poussé ses exploitations plus à l'ouest. Mais ce qu'elle en avait bûché du bois, tronçonné, des colonnes de fibre, et broyé, des tonnes et des tonnes de matière ligneuse : ils tracent une route principale, puis des routes secondaires qui sont perpendiculaires à cette route principale, et enfin des chemins 
soit en vigueur semble être celle de la propriété privée, sauvegardée par les habitants eux-mêmes. La police, en effet, n'est pas présente sur le territoire et lorsque les Autochtones causent des désordres, on dit qu'il y règne la loi du Far West (CB, 26 et 29). Le village, en outre, a été privatisé dans le cadre d'une conversion économique de la zone, précédemment réservée au déboisement pour l'implantation d'usines de papier. Sa relance se fonde sur une propagande publicitaire qui, de Montréal, atteint les États-Unis d'Ámérique :

\section{POURVOIRIE GRANDE-OURSE}

PARADIS DE CHASSE ET PÊCHE

LE CONFORT DE LA VILLE EN PLEINE FORÊT (CB, 19)

Une frontière bien visible, le chemin de fer, définit les limites entre le territoire des Amérindiens et celui des Blancs, entre nature et culture. Des fenêtres de la pourvoirie du village, qui se trouve dans une position stratégique, on aperçoit, après le restaurant, le passage à niveau et la route principale qui serpente vers le nord, en direction de l'hôtel. Un peu plus loin, au seuil de la forêt, près du Lac Légaré, s'élève le campement amérindien, que le remblai du chemin de fer sépare d'une tourbière, sorte de no man's land (CB, 22 et 195). Au nord, le cimetière, un dépôt de fercailles et une vieille usine de papier devenue inactive soulignent la paralysie économique du village, tandis que le reboisement spontané annonce un retour à la sauvagerie $(C B, 74)^{4}$.

tertiaires qui sont perpendiculaires aux routes secondaires. Et puis ils coupent, tondent bien ras, tout autour.» $(\mathrm{CB}, 18)$

4 Étant immergé dans la nature, Grande-Ourse n'est desservi par le train que trois fois par semaine, ce qui l'éloigne davantage de la culture de la métropole. La distance spatiale donne ainsi lieu à un décalage temporel, comme le souligne le narrateur : «Un employé s'arrangeait toujours pour nous balancer un exemplaire du Jourmal de Montréal, roulé bien serré autour de la haute teneur en sordide et en 
L'administrateur de - la pourvoirie croit en la renaissance de Grande-Ourse, mais il ne s'y rend que de temps en temps et son point de vue ne semble pas ancré dans la réalité : «Du haut d'une tour à bureaux, au milieu de Montréal, ou de son patio dallé de pavé uni et équipé d'un barbecue volcanique, Grande-Ourse pouvait encore hii apparaître comme un reflet de ce village ressuscité, ce nid de nantis qu'il avait aperçu une nuit en songe sous son édredon.» ( $\mathrm{CB}, 47)$ Pendant son absence, Benoît et le Vieux surveillent attentivement le magasin, «la poule aux cufs d'or de la Pourvoirie» (CB, 27). Bien que le jour, le magasin soit fréquenté comme une place publique (CB, 55), la nuit il est isolé, une véritable barrière le protégeant de l'extérieur, comme l'explique Gilles Deschênes, le nouveau commis de la pourvoirie :

Le magasin général de la Pourvoirie était hermétique et incorruptible, hanté par l'obsession de la sécurité. Dans un pays où fractures et effraction tiennent souvent lieu de formules de politesse, une simple serrure ne suffit pas. Benoît et le Vieux m'avaient expliqué ça très tôt et ils paraissaient fiers de leur système. Benoit fit glisser la lourde barre de métal en travers du double battant et il poussa la porte devant moi. La nuit enveloppante se déploya sous mes pieds. Derrière mon dos, aussitôt, la bâcle reprit sa position initiale et l'ancien poste de traite se referma en grinçant sur lui-même. $(\mathrm{CB}, 14)^{5}$

scabreux de la vie métropolitaine, et pas plus frais que les prétendues primeurs qui avaient fait le voyage avec lui. Il trônerait sur la table plusieurs jours avant de recueillir les épluchures des pommes de terre ou de servir de linceul à un poisson.» (CB, 32)

5 Les mésaventures du chat Hérode, que le Vieux empêche de sortir, confirment l'existence d'une barrière entre le magasin et le monde extérieur : «La frontière séparant les jeux innocents de la vraie vie, là où saignent les blessures et règne la souffrance» ( $C B, 70)$. Une fois sorti, en effet, le chat est déchiré par deux chiens féroces à proximité du chemin de fer constituant la limite principale du village. 
Gilles observe attentivement la vie de Grande-Ourse et fait le récit de ses expériences nordiques à la première personne. $\mathrm{Ce}$ personnage, alter ego de Louis Hamelin, qui a effectivement travaillé dans un magasin de la Haute-Mauricie ${ }^{6}$, est également l'auteur d'une narration à la troisième personne reconstruisant un événement de douze ans : le meurtre d'un jeune Amérindien, Roméo Flamand, duquel on a accusé Gilles Boisvert, fils du gérant de l'hôtel. Comme nous aurons l'occasion de le montrer, l'homonymie du prénom, Gilles, et la parenté sémantique du nom de famille, Deschênes/Boisvert, sont fondamentales pour comprendre l'évolution du protagoniste-narrateur en contact avec la réalité autochtone.

Le récit principal est divisé en cinq chapitres, chacun associé à une fête dérisoire, comme le remarque Jacques Allard ${ }^{7}$ : le jour de Dollard, la Saint-Jean, The Independence Day, Noël en juillet et le Pow-wow. Le récit possède un style qui n'est qu'apparemment prolixe, caractérisé par des mots difficiles et des expressions propres du langage parlé. ${ }^{8}$ Le roman dans le roman, au contraire, écrit à la troisième personne, est concis et poétique, riche en jeux de mots. L'énigme qu'il contient, au sujet du meurtre de l'Amérindien, n'est résolue qu'à la fin, lorsque Gilles Deschênes confronte ses hypothèses à ce qu'il est en train de vivre. Il y a donc un récit au premier degré, celui de Louis Hamelin, qui, pour l'utilisation de la première personne, se confond avec celui du narrateur fictif, Gilles Deschênes. Celui-ci, à son tour, est l'auteur d'un récit au deuxième degré qui, ouvrant une enquête sur le passé, produit un autre récit au troisième

6 J.-P. Fortin, "Cowboy de Louis Hamelin", Spirale, avril 1993, no 123 , p. 14.

7 Jacques Allard, «La nuit amérindienne», Le Devoir, 17 octobre 1992, p. D-3.

8 J.-P. Fortin, op. cit., p. 14. 
degré.? Paradoxalement, ce demier est au présent, tandis que la narration principale est au passé et à l'imparfait. L'effet stylistique qui en découle, outre qu'il actualise les événements se rattachant au meurtre, est essentiellement celui de reléguer dans le passé le présent utopique de Grande-Ourse :

Grande-Ourse se débattait à l'extrémité de sa route comme un poisson au bout de la ligne. Le monde lui donnait parfois du fil, lui faisait miroiter des espoirs ténus, des promesses jamais tenues, et alors le village partait en peur, s'embarquait sur un autre bateau qui prenait l'eau. Le monde donnait du fil à Grande-Ourse mais on lui laissait de moins en moins de jeu. $\mathrm{La}$ tension était maintenue à la limite de la rupture en un test continuel. Le monde lui donnait du fil comme pour l'aider à se noyer. (CB, 73)

Si la vie du village est suspendue à un fil, c'est parce que ses habitants sont toujours en conflit entre eux : d'un côté les Blancs, avec leurs idéaux utopiques et affairistes, de l'autre les Amérindiens, dont la passivité et l'inactivité sont excessives. Ces derniers, qui gravitent constamment en parasites autour du magasin, font de gros achats de bière et de nourriture d'importation qui les endettent toujours davantage. Le Vieux, qui les traite avec mépris de "Kawiches o Boucanés, accepte de devenir leur créancier car il espère encaisser les chèques qu'ils reçoivent du gouvernement. Cependant, les Autochtones, qui se déplacent sans arrêt le long du chemin de fer, dépensent

9 uJe griffonnais des notes en cachette, car je sentais que la suite de l'histoire me précipiterait dans un rôle pour lequel je devais déjà me préparer.» $(C B, 49)$ "J'en profitais pour colliger dans un camet des observations, des détails, des idées pour cerner, contraindre ce qui m'échappait, comme le coffrage d'une galerie de mine ceint le centre noir qui s'enfonce.» $(\mathrm{CB}, 113)$ 
ailleurs l'argent de l'État et lorsqu'ils rentrent à Grande-Ourse, ils ne possèdent plus rien ${ }^{10}$.

Quelle différence avec le tourisme à stars and stripes qui, à partir du dernier week-end de mai, Fête de Dollard, procure au village un important apport de dollars américains! La célébration d'un héros controversé ${ }^{11}$ ouvre ainsi les portes à l'envahissement estival des États-uniens, qui se rendent à Grande-Ourse pour la chasse et la pêche. Le narrateur les désigne comme «les Américains" par antonomase et les caricature dès leur entrée en scène :

En face, soudain, un véhicule de marque Cherokee, tout à fait véloce sur ses quatre roues motrices, apparut au sortir du virage et, sans donner la moindre impression de vouloir ralentir, se dirigea droit sur nous [...]. La Cherokee, rendue de l'autre côté en un tournemain, conserva malgré son train d'enfer une

$10 \mathrm{Si}$ le chemin de fer, comme on l'a remarqué, sépare le territoire des Amérindiens de celui des habitants de Grande-Ourse, dans le train les frontières sont sous-entendues et souvent, les Autochtones doivent payer pour être tranquilles. «Il s'agissait d'une forme de ségrégation très peu appuyée, un doux apartheid qui paraissait aller de soi pour le personnel de bord. On n'avait pas besoin de première et de seconde classes : tout était subtilement suggéré. Les familles aborigènes, avec la mère prèsque toujours flanquée d'une flopée d'enfants et le mari plus souvent qu'autrement égayé par de récentes dévotions bachiques, formaient des groupes plutôt bruyants, susceptibles de troubler le repos des quelques voyageurs de commerce solitaires et sommeilleux dont on voyait les têtes dodeliner au-dessus des bancs, dans le silencieux wagon des Blancs.» (CB, 89-90)

11 Dollard des Ormeaux, militaire de l'époque de la NouvelleFrance, vainquit un groupe consistant d'Iroquois en 1660; cependant, sa conduite fut ambiguë et il semble qu'il eut des contacts avec les Anglais dans le but de trahir sa patrie. 
maitrise relative de la situation et alla s'immobiliser assez brutalement contre un arbre. Aussitôt, quatre types vêtus de kaki en jaillirent en rigolant. L'un d'eux braquait une caméra vidéo et se mit sur-le-champ à mitrailler la scène de l'accident. Les autres promenaient autour d'eux des inities défiantes, comme des éclaireurs flairant une embuscade. (CB, 166-167) $)^{12}$

Si "Cherokee», le nom d'une nation amérindienne, correspond aujourd'hui à une automobile tout-terrain étatsunienne, le titre du roman, Cowboy, est paradoxalement le sobriquet d'un personnage amérindien. Un glissement sémantique a privé ces termes de leur signification originaire, car une double déformation a eu lieu : d'une part, l'Autochtone et le conquérant des origines ont acquis des caractéristiques qui ne leur sont pas propres; de l'autre, s'étant transformés, ils ne sont plus authentiques.

Même s'il est du côté des Amérindiens, le narrateur souligne la déchéance de leur culture et en attribue la responsabilité principale aux "Américains), comme nous allons le montrer par la suite. Sa rencontre avec l'Autochtone est précédée par une apparition nocturne très suggestive : «Au-dessus de ma tête, mêlant sa limaille de notes à la nuée d'étoiles qui pointillait le velours noir, la constellation fugitive des oiseaux, retour de migration, attaquait le prélude de ma rencontre avec Cowboy.»

12 Les «Américains» craignent une embuscade? Cela est naturel si l'on songe qu'ils se sont rendus à Grande-Ourse pour s'approprier la faune et la flore. Dans une autre circonstance, toujours grâce à la caricature, on voit émerger leurs attitudes de conquérants : «lls s'avançaient, comme en pays conquis, en territoire ennemi, ils surveillaient leurs arrières, ils portaient des chapeaux à large bord et des verres polarisés, ils mâchonnaient de fins cigares et frissonnaient dans le matin, ils étaient Américains.» ( $C B, 78-79)$ 
$(C B, 14)$ Il s'agit d'outardes dont la migration en été, saison où se déroulent les événements narrés, est un indicateur de nordicité. Les Amérindiens sont étroitement associés à ces oiseaux, en raison de leur nomadisme; en outre, eux aussi se déplacent vers le nord. Le fait qu'ils apparaissent la nuit semble accentuer le mystère et l'énigme qui enveloppent leur univers culturel, dont les Blancs n'ont qu'une connaissance superficielle. "Je ne distinguai d'abord que leurs ombres étirées glissant vers moi à la surface du sol» $(C B, 15)$, explique Gilles en se référant aux Amérindiens. L'atmosphère est celle d'un western : «lls marchaient le long des rails et ils étaient trois, comme dans un film de Leone» ( $C B, 15)$, même si le portrait des trois personnages s'inspire de la parodie. Le premier, une mauvaise imitation de Bruce Lee sortie d'une empoignade de série $B$ » $(\mathrm{CB}, 15)$, est surnommé Karaté $\mathrm{Kid}$; le deuxième s'appelle Donald-les-Bras, à cause de son étrange façon de marcher en oscillant les poings; le troisième, enfin, possède une dénomination paradoxale, "Cowboy", révélant un choix narratif que Louis Hamelin a explicité dans une entrevue :

Cowboy : C'est d'abord un mythe, ensuite une image. Un mythe que nous nous sommes appropriés au Québec où nous avons une culture westem sans avoir d'histoire westem. Notre vrai cowboy à nous c'est le coureur des bois. Pour le roman, j'ai poussé ce raisonnement au bout appelant un Indien du Québec Cowboy. ${ }^{13}$

La deuxième apparition des Amérindiens est aussi chargée de significations symboliques. Cowboy et Karaté Kid tourmentent avec le feu une couleuvre emprisonnée dans un bocal. «Au fond, c'est la même chose qu'ils ont faite aux

13 M.-C. Girard, «Les mois de Louis Hamelin», Le Devoir, 17 octobre 1992, p. D-3. 
missionnaires...» (CB, 28), affirme l'administrateur. En réalité, dans l'imaginaire de l'auteur, le reptile ne se rapporte pas uniquement aux francophones, mais aussi aux anglophones. Dans la même entrevue, où il l'associe, en fait, au Québec, Hamelin décrit le Canada comme un noud de vipères, deux scorpions enfermés dans une bouteille». L'analogie avec la situation romanesque qu'on vient de décrire est surprenante. On a aussi l'impression que le narrateur suggère que les Amérindiens gravitent en parasites, à cause du paternalisme inexcusable du gouvernement du Canada autour du petit agglomérat de Grande-Ourse, où sont en vigueur les lois du Far West.

Dans l'ensemble, l'univers culturel autochtone est plongé dans une atmosphère dramatique. Parmi les vapeurs de la nuit boréale, par exemple, il y a les exhalaisons de naphta, que les Autochtones inhalent pour s'évader de leurs misères quotidiennes, ou les effluves d'alcool, qui leur permettent d'oublier momentanément l'aliénation et la passivité dont ils sont victimes. Une myriade de personnages contribue à l'illustration du choc culturel découlant de la rencontre avec les Blancs. Les Amérindiens ont généralement des noms illustres, rappelant le fameux condottiere de la tradition occidentale - le Grand Alexandre, César, l'amiral Nelson - ou des héros plus ou moins dérisoires de l'univers cinématographique états-unien, comme on l'a déjà remarqué dans le cas de Cowboy et de Karaté Kid. Cependant, il ne s'agit pas de héros, mais de perdants, qui illustrent la déchéance de la culture indigène face à ce que les Blancs appellent le progrès : le Grand Alexandre, constamment entraîné dans des crimes et des bagarres, ne fait qu'entrer et sortir de prison. César Flamand, le père de l'Amérindien assassiné douze ans auparavant, est un être frustré et abattu qui a un passé d'alcoolique. L'amiral Nelson, un enfant qui rêve de devenir ingénieur, se noie dans les eaux d'une rivière à cause d'un incident. La mort de ce petit Amérindien devient un leitmotiv de la narration, soulignant les insuccès de la jeunesse 
autochtone, qui se réfugie dans un paradis artificiel stérile, en marge de la société des Blancs :

Dans le carré clôturé tout proche, sous les sables, l'amiral Nelson boit de la bière pour les temps à venir. Il ne regarde plus les hydravions, ne frappe plus la martre et ne pense plus à l'université. De l'autre côté du lac, près de la petite tourbière où l'eau et le sol se mêlent intimement, sous une grande tente d'arpenteur, Cowboy et les autres ont encore reniflé un peu de naphta avant de se remettre à rêver ce qui leur reste à rêver. (CB, 329)

L'univers des femmes amérindiennes est également désagrégé et corrompu. Gisèle, l'ancienne fiancée de Roméo Flamand, porte maintenant sur son visage les traces d'une décadence irréversible : «ll y a sur sa face jaunie et alourdie l'expression d'une béatitude effrayante, une sorte de satisfaction démoniaque qui s'exprime par un sourire qui est aussi une grimace, un pied de nez à toute notion de beauté» (CB, 189). Son corps est devenu amorphe, à la suite de nombreuses grossesses dérivant de son intense activité sexuelle : «Sa chair disgraciée est grosse des malheurs de la terre.» (CB, 189) Ses enfants sont éparpillés le long du chemin de fer, chez des parents, car elle n'est pas en mesure de s'occuper d'eux. Ainsi la destinée de sa fille aînée, Salomé, semble désormais tracée :

Née orpheline de père, d'une mère rendue veuve à coups de fusil, labourée en groupe ensuite et un peu déboussolée depuis. Confiée à la garde d'un grand-père silencieux et mélancolique qui aimait cependant les enfants. Sa belle-mère, une soiffarde sympathique et invétérée. Ses frères et sœurs naturels dispersés à gauche et à droite le long du chemin de fer. (CB, 320) 
Le nom de la jeune fille, qui n'a que douze ans, fait songer à la malice du personnage biblique qui demanda la tête du Baptiste. Cependant, on a déjà remarqué que, dans le roman, la valence sémantique des noms propres est altérée. Ainsi, Hérode n'est qu'un chat féroce qui, une fois sorti de son monde, est dépecé sans pitié. La Saint-Jean n'est qu'une fête dérisoire, celle du Québec, qui se déroule dans un climat lugubre, après la mort du petit Nelson. Salomé ne pourra pas être protagoniste d'une affirmation positive de lindianité. Il est plutôt probable que sa beauté contribuera à la perdre : "dans dix ans, dans cinq ans et même bien avant, elle sauterait sur les hommes, elle aussi) $(\mathrm{CB}, 320) \cdot{ }^{14} \mathrm{La}$ jeune Amérindienne est amoureuse de Gilles, qui cherche cependant à préserver son intégrité. Dans un premier temps, il l'empêche de renifler le naphta, ensuite il résiste à ses charmes, même s'il sait bien qu'à l'avenir, Salomé fera sans doute la danse des sept voiles pour quelqu'un d'autre ${ }^{15}$.

C'est Salomé qui annonce à Gilles qu'on a retrouvé le corps de Cowboy noyé dans la tourbière, «les pieds dirigés vers le ciel et posés sur le film délicat qui sépare les deux mondes, comme s'il avait simplement voulu faire une petite promenade sous le décor, de l'autre côté du reflet» (CB, 366). Auparavant, l'Amérindien avait adopté la même position pour explorer les

14 Parmi les personnages féminins, il faut mentionner aussi Judith, une jeune Amérindienne qui, étant tombée amoureuse d'un Blanc, a vainement essayé de pénétrer de l'autre côté de la frontière. Son amant, après la naissance d'un garçon, l'a abandonnée à sa destinée. À présent l'enfant vit dans la réserve, chez une tante, tandis que la mère n'a pas honte d'offrir son corps au gros Moreau, le propriétaire du restaurant, qui finit par chasser sa femme pour accueillir l'Amérindienne chez lui.

15 Dans les pages finales du roman, le narrateur écrit que Salomé, comme beaucoup d'autres Amérindiens, a abandonné Grande-Ourse pour Montréal, où elle vit avec une cousine qui est danseuse nue. Peut-être bientôt suivra-t-elle son exemple? 
eaux du Lac Légaré à la recherche d'un trésor légendaire caché dans une bouteille. Gilles rappelle ainsi cette circonstance : "Je m'adressais maintenant à une paire de jambes dressée à la verticale dans l'obscurité.» (CB, 177) Le suicide de Cowboy semblerait un aveu de sa comuption, car l'argent qu'il disait avoir trouvé dans le lac, en réalité, avait été volé au touriste américain Crazy Sam. Maintenant la boue recouvre son corps, et la tourbière où il a voulu se noyer est devenue l'emblème de l'altération de la terre et de l'eau, des éléments que l'Amérindien maîtrisait lorsqu'il était "puo». "Cowboy, c'était aussi le héros aquatique, le phénomène amphibie. Il pouvait rester des minutes entières sous l'eau, à attendre la déplétion de sa

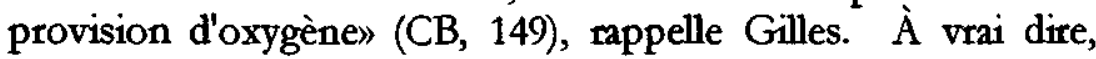
dans ses plongées, il y avait déjà une préfiguration du drame dont il serait victime :

La fluidité de ses mouvements avait quelque chose de magique qui me rappela la séquence d'Apocalypse Now où Martin Sheen, figé dans une lenteur sensuelle et eidétique, se glisse pouce par pouce hors de l'eau bourbeuse avec, en fond de toile, les danses paroxystiques qui entourent le sacrifice d'une tête de bétail. (CB, 176)

Il faut aussi rappeler que la tourbière coïncide avec le no man's land entre le monde des Autochtones et celui des Blancs. Le rôle narratif de l'Amérindien, en fait, est celui d'intermédiaire entre les deux espaces. En quête des valeurs profondes de l'amitié, Cowboy a aidé Gilles à traverser la frontière du côté de la sauvagerie pour l'introduire dans l'univers autochtone. En même temps, il a adopté des traits culturels propres de l'autre versant qui ont déterminé sa dénomination paradoxale :

Ses amis l'appelaient Cowboy et il était Indien d'Amérique. Il avait hérité de ce surnom un soir qu'on 
l'avait vu venir le long de la voie ferrée, sa silhouette efflanquée se découpant sur le couchant dans une longue capote western dont les plis battaient contre ses bottes de cuir. (CB, 13)

Parmi ses accessoires, une Winchester 30/30, «'antique carabine des cowboys» (CB, 102), mais aussi une machette qu'il a mise dans les mains de Gilles lors de leur première rencontre $(\mathrm{CB}, 16)$. Quant à ses goûts alimentaires et musicaux, le poulet Tropicana et la chanson La Isla bonita, reflètent son aliénation et peut-être un rêve d'évasion dans le sud. Le morceau "Vivre dans la nuit», une autre de ses chansons préférées, suggère le mystère qui l'a enveloppé et que le narrateur n'a pas été en mesure d'éclaircir, l'Amérindien ayant toujours eu une attitude rigide et impassible : «Ses pommettes altières et ses yeux de bouddha ressortaient parmi des linéaments d'une irréprochable symétrie, capables de réduire tout sentiment inopportun à un inoffensif pli de la peaus ( $C B, 16)$. Cowboy avait aussi un sourire de sphinx nuancé de mélancolie, comme le rappelle le narrateur : "J'observais Cowboy à la dérobée, ce Cowboy secret avec ses grosses joues pompant notre joie commune. La lucidité de la drogue me faisait pénétrer dans l'ombre de ce visage de pleine lune où la mélancolie et la folie de sa race faisaient bon ménage.» $(C B, 95)$ Gilles essaie de s'expliquer ainsi son attirance pour Cowboy:

Car il ne m'attirait pas tant comme individu que comme exemplaire produit de sa culture. Notre amitié n'avait de sens qu'au pluriel, c'était une amitié de fond, indifférente aux détails dont se composent habituellement les affinités. C'est l'Indien que j'aimais en Cowboy. Je me rendais parfaitement compte de cette discrimination positive. L'acceptation de l'opacité inaliénable de l'autre me paraissait le seul partage possible entre nous. (CB, 239) 
En généralisant, on pourrait dire que l'Amérindien demeure impénétrable pour le Québécois, malgré les affinités existant entre eux, telle que l'aversion mal cachée pour la culture étatsunienne. Au-delà des apparences, Cowboy a été profondément lié à son indianité et a refusé de se faire guide pour les touristes américains. Il ne leur a même pas vendu la fourrure de l'ours qu'il a tué d'un seul coup avec sa 30/30. Les Amérindiens de la tribu des Algonkins, en réalité, ne peuvent pas vendre l'ours, animal qu'ils considèrent comme un ancêtre mythique. Cowboy a donc offert à Gilles de la viande et une dent de l'ours, échange culturel qui a renforcé leur amitié. Lors de ses funérailles, Gilles fait glisser la dent dans la fosse, car l'objet a perdu sa signification originelle, en outre il mange la viande qu'il a gardée dans le congélateur jusqu'à ce moment-là. Il intériorise ainsi ses liens d'amitié avec l'Amérindien, même si ces derniers sont inscrits dans la perte. L'anthropologue Sylvie Vincent a donc raison lorsqu'elle observe que l'Autochtone est destiné à disparaître même s'il est perçu positivement - Gilles a fait allusion à «une discrimination positive». ${ }^{16}$

Le drame de Cowboy se rattache aux événements d'il y a douze ans concemant le meurtre de Roméo Flamand, pour lequel a été condamné le fils du propriétaire de l'hôtel, Gilles Boisvert. Comme nous l'avons déjà dit, la narration que Gilles Deschênes insère en italique dans le récit principal essaie de reconstruire les circonstances du meurtre de l'Amérindien. Cependant, au fur et à mesure qu'il écrit, le narrateur arrive à s'identifier avec Gilles Boisvert. Dans les pages finales de ses notes, écrites à la troisième personne, le lecteur voit ainsi apparaître un «je» qui s'interroge sur ses intuitions : "comment

16 Sylvie Vincent, «De la nécessité des clôtures. Réflexion libre sur la marginalisation des Amérindiens", Anthropologie et Société, vol. X, no 2,1986 , pp. 75-83. 
sais-je tout cela? La réponse est encore une fois assez simple : parce que Gilles Boisvert, c'est un peu moi.» $(\mathrm{CB}, 410)$

Une autre illumination du narrateur concerne l'ambiguité de Jacques Boisvert, aux yeux duquel il est comme un fils. Même lorsqu'il découvre que Gilles est devenu l'amant de sa femme, le propriétaire de l'hôtel ne semble pas particulièrement jaloux: "Ce jeune commis qu'ils ont engagé, à la Pourvoirie. Me rappelle le mien, tiens, il est pareil. Brigitte le trouve de son goût” (CB, 329), se bome-t-il à observer. Les Amérindiens, de leur côté, n'aiment pas l'amitié entre Gilles et Boivert, car ils pensent que ce demier est responsable de la mort de Roméo. Mais qui a tué l'Amérindien? Certains pensent que le fils a été condamné à la place du père et que Jacques Boisvert n'était pas loin de l'hôtel le soir du meurtre. En outre, Crazy Sam, un riche touriste étatsunien, avait donné mille dollars américains à Gilles Boisvert afin qu'il tue Roméo mais, le jeune homme manquant de courage pour commettre le meurtre, Jacques Boivert lui-même avait assommé l'Amérindien.

Le père, qui au sens figuré renvoie au Québécois des origines, a frappé d'un coup mortel la société amérindienne, à présent aliénée et sans ressources. La complicité des Américains a été fondamentale : le personnage de Crazy Sam fait songer, en effet, à Uncle Sam, le symbole des États-Unis et de leur pouvoir économique. On peut aussi remarquer que le meurtre a mûri dans un climat de jalousie autour de la beauté physique de l'Autochtone, le charme de Roméo et Gisèle suscitant l'envie et le désir de Gilles Boisvert et des touristes de l'hôtel :

Et Gisèle qui se montre à l'hôtel, switée par ce paon à la peau cuite, tendue sur des muscles en saillie. La fière allure de ce Flamand, ses épaules amples, sa denture de palefroi, un étalon, de la crinière aux talons. Gilles est jaloux, forcément. (CB, 153-154, en italique dans le texte) 
Avec le recul, Jacques Boisvert a compris l'attirance que son fils éprouvait pour la belle Amérindienne, et c'est peut-être pour cela qu'il s'est montré indulgent envers Gilles Deschênes, son fils d'adoption. Avant la mort de Cowboy, par exemple, lorsque les Blancs étaient en train de fêter à l'hôtel un étrange Noël en juillet, il a permis que Gilles et Brigitte se rendent à l'autre fête : celle du campement autochtone. Il a même offert un cadeau symbolique au jeune homme : une poupée amérindienne, de celles qu'on trouve dans les magasins de souvenirs, «la princesse Pocahonta en personne, c'est-à-dire en pacotille.» (CB, 355) Malheureusement il était tard, le folklore demeurant le seul vestige de la culture autochtone. La conclusion de l'aventure nordique de Gilles confirme la décadence caractérisant les sociétés amérindiennes après l'arrivée des Blancs. Lors des funérailles de Cowboy, Gilles outrepasse la frontière entre les deux mondes pour se rendre à la réserve, où les misères morales et matérielles des Autochtones lui font éprouver un sentiment de culpabilité :

Les mesures du temps s'occultaient. Un seul repère restait valable au sein de cette assommante succession : la nuit. Tout le monde y vaguait l'âme en berne, s'y meurtrissait et s'y maudissait, s'y vengeait, s'y mourait d'amour et s'y morfondait de haine. Et le jour, tout s'écroulait de nouveau et un silence de mort régnait en maitre sur les cabanes croupissantes. Il ne restait que moi pour avancer au hasard, les bras le long du corps et la tête basse sous la lumière drue, trop coupable de tous les crimes pour jamais sombrer dans l'apaisement. (CB, 385)

Par ailleurs, le racisme des Amérindiens à l'égard des Blancs met en danger sa vie même. Le Grand Alexandre, qui n'a jamais approuvé son amitié pour Cowboy, veut le battre. Il fait sien le ressentiment de César Flamand, lequel accuse publiquement le commis de la pourvoirie d'être Boisvert, l'assassin de son fils. 
Gilles ne peut pas prouver son identité, car on lui a volé ses papiers :

[...] en me dépouillant de mes affaires, on m'avait volé mon identité. Privé de ma définition sociale et de tout recours immédiat, je me retrouvais à la merci de cette meute instable. Je pouvais bien m'appeler Gilles Boisvert, après tout. ( $\mathrm{CB}, 408)$

Cela témoigne de l'évolution du héros, qui s'est tout à fait identifié avec Gilles Boivert. La situation devient dramatique pour lui. Seuls Karaté Kid, Salomé et quelques autres le défendent. Entre-temps, l'arrivée de Jacques Boivert attire l'attention des présents. Quand l'hydravion du propriétaire de l'hôtel s'écrase comme un météore avec Crazy Sam dans le cimetière de la réserve, Gilles Deschênes comprend par intuition ce qui s'est passé le jour du meurtre :

Cette nuit-là, un père nia un fils, et tout s'est passé ensuite comme si ce fils nié m'avait délégué à GrandeOurse pour forcer le père à expier sa part de la faute. Je suis devenu le rédempteur d'une trinité assassine. $\left(\mathrm{CB}, 413\right.$, en italique dans le texte) ${ }^{17}$

17 On a l'impression que le demier geste de Jacques Boisvert est prémédité, de même que celui de Cowboy. Lorsqu'ils aperçoivent le Beaver en difficulté survolant la réserve, les Amérindiens remarquent qu'il pourrait se poser n'importe où, car la zone est entourée d'eau. En fait, l'appareil fait des manoeuvres dangereuses que Gilles observe attentivement : "Je me contentai de me baisser et, relevant la tête, j'eus ma dernière vision de Jacques Boisvert, pilote de brousse et dur de dur des durs de Grande-Ourse : tourné vers moi, et si proche que je pouvais parfaitement discerner le blanc de ses yeux, il m'adressait un invraisemblable salut. L'hydravion plongea vers la colline qui dominait la presqu'île et, comme pour une ultime provocation, alla 
L'épilogue du roman est brusque et soudain. Grande-Ourse brûle car lun des frères de Salomé, en jouant avec des allumettes, a causé un incendie. Le village survit, mais les relations entre les deux communautés ne seront plus les mêmes, car les Autochtones considèrent Grande-Ourse, un lieu maudit, et qu'ils ont commencé à le déserter pour se diriger phus au nord. Voilà la signification première de la nordicité dans le roman : la néo-colonisation du territoire de la part des Québécois entraîne la disparition des Amérindiens, destinés à migrer jusqu'à ce qu'il n'y ait plus de nord pour eux. Le campement autochtone n'est désormais qu'un vague souvenir et le narrateur, avant de rentrer à Montréal, accomplit son demier pèlerinage à la tourbière dans laquelle Cowboy s'est suicidé, et où il ne reste qu'un "trou noin». On peut en déduire qu'une partie de la matière qui constituait le petit agglomérat de Grande-Ourse - autochtone - a été absorbée par la terre et annulée. Même si le Québécois a assumé le poids de ses fautes, à cause des avatars de la colonisation son amitié avec l'Amérindien s'est inscrite dans la perte.

s'écraser au beau milieu de ce cimetière grillagé dont au moins un lopin était fraîchement retourné.» $(\mathrm{CB}, 412)$ 